\title{
El programa académico de servicio social de medicina en la modalidad blended-learning: estudio comparativo con el método tradicional
}

Mc. Carolina Zazueta Robles

Dra. María Guadalupe Soto Decuir

soto.decuir@gmail.com

Mte. Luis Alberto González García

drlagg@gmail.com

Mte. Cesar Roberto Jiménez Martínez.

crji_03@uas.edu.mx

Universidad Autónoma de Sinaloa

Facultad de Medicina

Recibido: 05 de mayo del 2020

Aceptado: 05 de junio del 2020

\section{Resumen}

En el proyecto "Implementación del programa académico de servicio social en medicina mediante la modalidad blended- learning" se propuso comparar dicha implementación con la modalidad tradicional, con el fin de incrementar el nivel de aprendizaje de los médicos brigadistas por vinculación universitaria de la Facultad de Medicina de la Universidad Autónoma de Sinaloa. El objetivo fue mejorar el aprendizaje de los médicos brigadistas de servicio social asignados a vinculación universitaria. El material se diseñó siguiendo el modelo ASSURE que tiene sus raíces en el constructivismo, parte de las características concretas del estudiante, de sus estilos de aprendizaje y fomenta a la vez la participación y compromiso. Para el logro del objetivo, se trabajó con médicos brigadistas que realizaron su servicio social por vinculación universitaria, durante agosto de 2018 a julio 2019. Se seleccionó el diseño experimental (grupo experimental y un grupo control), con un enfoque cuantitativo, de tipo descriptivo y corte transversal. Los participantes fueron seleccionados mediante el muestreo probabilístico de manera aleatoria. Se aplicó el pretest y postest al grupo control y experimental. El grupo experimental recibió el tratamiento. Se analizaron dos variables de interés, la variable independiente: blended-learning y la variable dependiente: nivel de aprendizaje. Se aplicó el estadístico para pruebas relacionadas de $t$ de student para comprobar la hipótesis nula: Al implementar el programa académico de servicio 
social en modalidad blended-learning se incrementa el nivel de aprendizaje en los médicos brigadistas de servicio social por vinculación universitaria. Se concluye que la implementación del programa académico de servicio social de medicina en la modalidad blended-learning mejoró el aprendizaje de los médicos brigadistas asignados a vinculación universitaria. El trabajo realizado corrobora el apoyo de integrar las TIC como estrategias y recursos didácticos al proceso educativo.

Palabras clave: Aprendizaje, TIC, Blended-learning, Servicio Social.

\section{The academic program of medical social service in the blended- learning modality: comparative study with the traditional method}

\section{Summary}

In the project "Implementation of the academic program of community service in medicine through the blended-learning modality", it was proposed to compare this implementation with the traditional modality, in order to increase the level of learning of medical brigade members by university connections of the Faculty of Medicine of the Autonomous University of Sinaloa. The objective was to improve the learning of the brigade doctors of social service assigned to university connections. The material was designed following the ASSURE model that has its roots in constructivism, part of the specific characteristics of the student, of their learning styles and at the same time encourages participation and commitment. To achieve the objective, we worked with medical brigade doctors who carried out their community service through university ties, during August 2018 to July 2019. The experimental design (experimental group and a control group) was selected, with a quantitative, descriptive type approach and cross section. Participants were selected by random probability sampling. The pretest and posttest were applied to the control and experimental group. The experimental group received the treatment. Two variables of interest were analyzed, the independent variable: blended-learning and the dependent variable: learning level. The statistic for related tests of student $t$ was applied to test the null hypothesis: When implementing the academic program of community service in blended-learning modality, the level of learning is increased in the brigade doctors of community service by university affiliation. It is 
concluded that the implementation of the academic program of medical community service in the blended-learning modality improved the learning of the brigadista doctors assigned to university connections. The work carried out corroborates the support of integrating ICT as strategies and didactic resources to the educational process.

Keywords: Learning, ICT, Blended-learning, Community Service.

\section{Introducción}

La finalidad de este estudio es comparar la implementación del programa académico de servicio social establecido por la Secretaria de Salubridad y Asistencia (SSA) en la modalidad blended-learning con la modalidad tradicional, para mejorar la calidad de la enseñanza en los médicos brigadistas por vinculación universitaria. Los motivos que llevaron a desarrollar esta investigación fueron los siguientes:

- Los médicos brigadistas asignados a la modalidad de vinculación universitaria llevan a cabo el programa académico en la modalidad tradicional y los propuestos a la modalidad comunitaria o de investigación en instituciones públicas como el Instituto Mexicano del Seguro Social (IMSS) y la Secretaria de Salubridad y Asistencia, tienen un seguimiento estricto y riguroso del programa académico, mediante sesiones presenciales, con exposiciones de temas y revisión de casos clínicos.

- En el servicio social del área de la salud, el médico brigadista como estudiante en formación necesita contar con el respaldo de su institución educativa, no solo en el cumplimiento, sino en su formación académica, lo cual motivó a llevar a cabo la aplicación y evaluación de un material del programa académico de servicio social en la modalidad blended-learning para apoyar a estos sujetos a mejorar su aprendizaje. Para ello, se emprendió una investigación que permitiera el diseño del material y que tuviera la finalidad de contribuir a la mejora del aprendizaje y, por ende, a su labor profesional.

- Las presentes líneas de enseñanza reflexionan en fomentar el aprendizaje a lo largo de la vida por medio de las tecnologías; en este sentido y considerando que existe un problema en el cumplimiento del programa académico de servicio social en los médicos brigadistas asignados a la modalidad de vinculación universitaria, se propuso 
elaborar un material educativo que sirviera para incrementar su nivel de conocimiento.

- La idea de trabajar con las Tecnologías de la Información y la Comunicación (TIC) se debe a sus características (asequibles, innovadoras, creativas), ya que se consideran elementos idóneos para favorecer el aprendizaje; las TIC propician autonomía y autoaprendizaje; para ello el material se diseñó en la plataforma Moodle.

La Dirección General de Calidad y Educación en Salud (DGCES) y la Secretaria de Salud y Asistencia, han acordado generar nuevos procesos educativos para cumplir con la competencia y calidad del ejercicio profesional de los médicos brigadistas; es por ello que se propuso implementar el diseño de una estrategia mediante sesiones presenciales con exposiciones y discusiones de casos clínicos acordes al programa académico de servicio social y temas de actualidad, junto con el uso de las TIC como son actividades de formación, retroalimentación y evaluación que contribuyan al desarrollo de su labor profesional.

La sociedad demanda profesionales competentes, capaces de actuar con humanismo y sentido social, desenvolverse con autonomía y desarrollar el aprendizaje autorregulado y a lo largo de la vida. Ser un médico profesional con la capacidad de autorregular su aprendizaje por medio de las TIC, le otorga mayor ventaja en su dimensión formativa y profesional respecto a quienes no las dominan. Dado lo anterior, en este estudio se trabajó con las TIC y el aprendizaje como posibles variables.

La incorporación de las TIC a la educación apoya nuevas formas de aprendizaje; sin embargo, sólo refuerzan y promueven la innovación cuando se insertan en una dinámica de innovación y cambio (Coll, Mauri \& Onrubia, 2008, p. 97). El desarrollo de entornos de enseñanza y aprendizaje para esta nueva era, es una tarea compleja debido a la cantidad, calidad y rapidez de los cambios (Mauri \& Onrubia, 2008, p. 133). Los métodos de enseñanza no están adaptados a los estudiantes actuales, hay insuficiente preparación docente con relación al uso de estrategias y recursos tecnológicos para modificar su labor profesional.

Ante el interés de mejorar el aprendizaje integrando las TIC al proceso educativo, el conflicto que poseen los docentes en incorporarlas y la idea errónea que tienen al aplicarlas, se diseñó, implementó y evalúo un material educativo en un entorno virtual de aprendizaje 
basado en el análisis de casos clínicos y la resolución de problemas para el programa académico de servicio social de medicina. Diferentes autores señalan que incorporar las TIC a la educación universitaria es una posibilidad para que los estudiantes adquieran y desarrollen las competencias de aprendizaje a lo largo de la vida (Coll \& Monereo, 2008; Cáceres, 2015; López de la Serna, 2016).

En la implementación del programa académico del servicio social de la SSA, el eje central es el estudiante-médico como futuro profesional que debe actualizarse continuamente, no obstante, el apoyo de médicos profesionales del área simbolizó un refuerzo como expertos en su disciplina. Lo anterior obligó a trabajar de manera multidisciplinaria y encontrar espacios para dialogar y reflexionar en grupo, analizar en conjunto sobre el rol de estudiante de los médicos brigadistas con profesionales del área de medicina y expertos en tecnología educativa.

Integrar las TIC al proceso educativo exige que los estudiantes realicen diferentes acciones, entre ellas, autorregular su aprendizaje y planear sus actividades, lo cual significa controlar la procrastinación, y desarrollar habilidades críticas y metacognitivas (Martínez \& Rabanaque, 2008). Según Cobo (2009) el hecho de saber usar las TIC no basta para formar una sociedad del conocimiento, lo importante es utilizarlas como medios necesarios para dar a conocer los conocimientos. En cualquier área de conocimiento, "Los estudiantes, al salir de una carrera universitaria, tienen la necesidad de seguir aprendiendo para mantenerse actualizados en su contexto, por lo que debe haber un aprendizaje continuo que converja en la vida profesional" (Soto, 2014)

\section{Diseño}

El estudio se planteó a partir de la siguiente pregunta: ¿Cómo implementar el programa académico de servicio social en la modalidad blended-learning para incrementar el nivel de aprendizaje en los médicos brigadistas por vinculación universitaria de la Facultad de Medicina de la Universidad Autónoma de Sinaloa? A partir de lo anterior, se estableció el diseño, cuyo objetivo es mejorar el aprendizaje de los médicos brigadistas de servicio social asignados a vinculación universitaria. Bajo la hipótesis nula: Al implementar el programa 
académico de servicio social en modalidad blended-learning se incrementa el nivel de aprendizaje en los médicos brigadistas de servicio social por vinculación universitaria.

El material se diseñó siguiendo la implementación del modelo ASSURE que tiene sus raíces en el constructivismo, parte de las características concretas del estudiante, de sus estilos de aprendizaje y fomenta a la vez la participación y compromiso (Belloch, 2013). Este modelo guio el proceso instruccional de un curso blended-learning de una unidad de aprendizaje del programa de servicio social de la Licenciatura en Médico General, se planearon actividades que complementaran su quehacer diario en su práctica médica, considerando que los médicos brigadistas de hoy en día se encuentran inmersos en el uso de la tecnología, se pretendió que con la implementación de la modalidad virtual el estudiante se motive y abone de manera favorable a su proceso de aprendizaje.

\section{Metodología}

Para el logro del objetivo se seleccionó el diseño experimental, los participantes fueron seleccionados al azar, se contó con un grupo experimental y un grupo control. Se aplicó el pretest simultáneamente al grupo control y experimental. El grupo experimental recibió el tratamiento; por último, se les administró el postest a ambos grupos.

Se analizaron dos variables de interés, la variable independiente: blended-learning conceptualizada como la modalidad de aprendizaje semi presencial, mixta o bimodal, también llamado híbrida. Combina la modalidad presencial y la virtual fusionando las ventajas de ambas en pro de los estudiantes; y la variable dependiente: nivel de aprendizaje como la capacidad de mejorar el desempeño académico basándose en la experiencia de integrar las TIC al proceso educativo.

El presente estudio se realizó bajo el enfoque metodológico cuantitativo, de tipo descriptivo y corte transversal. Para llevar a cabo la investigación se trabajó con médicos brigadistas de la Facultad de Medicina de la Universidad Autónoma de Sinaloa que realizaron su servicio social en vinculación universitaria, en el periodo de agosto 2018 a julio 2019. La matrícula de alumnos de servicio social de la Facultad de Medicina para el ciclo mencionado fue de 669 alumnos. De los cuales el 35 por ciento estaba asignado a plazas por vinculación 
universitaria distribuidos en la zona centro del estado de Sinaloa, de dicho porcentaje se excluyeron las plazas hospitalarias, porque en esta modalidad sí se lleva a cabo el programa académico para los médicos brigadistas en hospital, por lo que la población se redujo a 138 alumnos.

La muestra se eligió por medio del muestreo probabilístico, que consiste en tomar de manera aleatoria a los participantes a partir de un marco muestral, en donde todos los participantes tienen una probabilidad conocida y distinta a cero de ser escogidos. PérezTejeda (2008) menciona que:

La selección de la muestra se realiza con base en fundamentos de la teoría de probabilidad, lo cual permite hacer una evaluación objetiva de los resultados y, por ende, se está en posibilidad de conocer el grado de precisión y confianza de los mismos. Por tanto, en el muestreo probabilístico, una vez definida la población de estudio, configurado el marco de muestreo y definida la forma de selección, la conformación de la muestra no depende de los criterios selectivos o preferencias del investigador (p.185)

De los 138 médicos-brigadistas asignados a la modalidad de vinculación universitaria, se calculó el tamaño mediante la fórmula de población finita con un nivel de confianza del 95 por ciento, el resultado fue de 57 estudiantes. Tanto para el grupo experimental como el grupo control se consideró la misma cantidad. El trabajo de campo se llevó a cabo durante 6 semanas, correspondiente a los meses de marzo y abril del 2019. El grupo control desarrolló actividades de forma tradicional (consulta general, brigadas comunitarias y promoción a la salud). El grupo experimental en la modalidad blendedlearning.

\section{Instrumentos y técnicas}

Con base en el modelo ASSURE (6 etapas), se realizó el diseño instruccional, el cual incluye la planeación de actividades por semana (6 semanas con 1 sesión presencial de 2 horas cada una y las actividades de las sesiones en línea). Las sesiones presenciales se llevaron a cabo 
en las instalaciones de la Facultad de Medicina de la UAS, y las sesiones en línea cada estudiante las podía realizar en el lugar y hora que le favoreciera.

\section{Analizar las características del estudiante.}

Antes de comenzar cualquier curso, se deben conocer las características generales de los estudiantes, las capacidades específicas de entrada y los estilos de aprendizaje.

\section{Establecer los objetivos de aprendizaje.}

Se desarrolló en apego al programa académico para los médicos pasantes en servicio social comunitario con enfoque en medicina familiar que establece la Secretaría de Salubridad y Asistencia como obligatorio a partir de agosto de 2017. El propósito de enseñanza de dicho programa es el estudio interdisciplinario del proceso de saludenfermedad en las familias y sus integrantes en su contexto biológico, social y psicológico con un enfoque de riesgo y una atención continua integral.

\section{Selección de estrategias, tecnologías, medios y materiales.}

Se eligieron las tecnologías y los medios adecuados para posteriormente decidir con qué materiales y estrategias trabajar. Se utilizó la plataforma Moodle de la Facultad de Medicina para complementar las sesiones presenciales. Los recursos que sirvieron de apoyo para lograr el propósito del curso dentro de la plataforma consistieron en texto, videos, imágenes y herramientas de la web 2.0.

\section{Organizar el escenario de aprendizaje.}

Se creó un escenario que propiciara el aprendizaje de manera activa y colaborativa y que conllevara a la gestión de su propio aprendizaje y en la autorregulación académica, en este caso mediado por el uso de las tecnologías. De acuerdo a lo anterior se creó el escenario para la unidad de aprendizaje correspondiente al estudio de Atención del niño y del adolescente enfermo. 


\section{Participación de los estudiantes.}

Es necesario que el estudiante participe de manera activa para lo cual se requiere gran compromiso de su parte, motivado por el guía o facilitador del curso. Las actividades planeadas se distribuyeron respetando los tiempos asignados para la unidad, cada módulo se estructuró de la siguiente manera:

\section{Primera semana: Encuadre}

El encuadre fue el primer acercamiento de la facilitadora con los participantes del curso, para lo cual fueron citados de manera presencial en las instalaciones del Centro de Entrenamiento Virtual y Tecnologías Educativas (CEVyTE) de la facultad, con la finalidad de dar a conocer la dinámica de trabajo. Los recursos utilizados en la plataforma para esta semana fueron: Cuestionario para el pretest, donde el estudiante respondió una serie de preguntas sobre conocimientos previos referentes a la unidad de aprendizaje Atención del niño y del adolescente enfermo, sirvió de referencia para dar cuenta del nivel de dominio de los temas a desarrollar en el curso.

\section{Segunda semana: Infección de vías aéreas respiratorias}

En la sesión presencial se analizó el caso clínico infección de vías respiratorias. La sesión en línea consistió en foros, se utilizó para socializar y discutir el caso. La Tarea de esta semana consistió en elaborar un mapa conceptual a partir de las lecturas compartidas en el apartado de recursos.

\section{Tercera semana: Enfermedades diarreicas y parasitosis intestinal}

El tema de análisis en la sesión presencial fue las enfermedades diarreicas, la dinámica de las sesiones en línea fueron las siguientes: Tarea: los estudiantes elaboraron un diagrama de flujo sobre enfermedades diarreicas, a partir de los conocimientos adquiridos en la sesión presencial y con la revisión de las lecturas del apartado de recursos. El Cuestionario: se utilizó para evaluar los conocimientos adquiridos sobre el tema parasitosis intestinal. 


\section{Cuarta semana: Alteraciones del estado nutricional}

Después de la sesión presencial, donde se analizó el caso clínico, se utilizaron para las sesiones en línea Recursos web 2.0 para la resolución del crucigrama Alteraciones del estado nutricional, elaborado con la herramienta HotPotatoes. También se asignó una Tarea, donde los participantes realizaron una reflexión después de ver el video Malnutrición, principal causa de malnutrición y obesidad.

\section{Quinta semana: El niño atópico y enfermedades exantemáticas}

Durante esta semana por medio una videoconferencia se impartió el tema, se estableció una actividad para evaluar los contenidos correspondientes al niño atópico y enfermedades exantemáticas. Se utilizó el Cuestionario para evaluar los contenidos del tema el niño atópico y enfermedades exantemáticas, los estudiantes respondieron preguntas de imagen y texto con la finalidad que identificaran las lesiones características de las principales patologías que cursan con exantema.

\section{Sexta semana: evaluación}

En esta semana los participantes respondieran el postest y realizaran la evaluación general del curso

\section{Evaluación y revisión de la implementación y resultados del aprendizaje}

Representa la última etapa del proceso. Aunque se realizó la evaluación periódica de los aprendizajes de los participantes, una vez finalizada la intervención es conveniente evaluar la eficacia y satisfacción del curso. Se utilizaron cuestionarios para evaluación de conocimientos previos sobre la temática del curso (pretest), para cada una de las actividades se utilizaron rúbricas de evaluación, al final se realizó la evaluación final de contenidos (postest), así como evaluación general del curso (cuestionario de satisfacción). 


\section{Análisis de datos}

Para el análisis de datos se utilizó Microsoft Excel y el programa estadístico SPSS. Se analizaron frecuencias, porcentajes y muestras relacionadas de los grupos de control y experimental. Para el análisis estadístico de los niveles de aprendizaje se utilizó la prueba $t$ de Student para dos muestras relacionadas, dadas las características del diseño de investigación, el tipo de prueba estadística para el diseño de investigación fue paramétrica.

\section{Resultados}

Los médicos brigadistas de servicio social (grupo experimental) realizaron el pretest Atención del niño y del adolescente enfermo, sobre los contenidos que se abordarían en el curso de forma presencial en el Aula Virtual. Después se trabajó con el grupo de control, indicándoles la manera de realizarlo para tener el punto de comparación.

El grupo experimental llevo a cabo actividades presenciales con casos clínicos de los siguientes temas: infección de vías aéreas superiores e inferiores, enfermedades diarreicas y parasitosis intestinal, alteraciones del estado nutricional, niño atópico y enfermedades exantemáticas de acuerdo con los contenidos del programa. Las actividades del Aula Virtual en este periodo (marzo-abril 2019) consistieron en foros de discusión que tuvieron una participación de $83.7 \%$, el desarrollo de mapas conceptuales $77.7 \%$, diagramas de flujo $80.7 \%$, cuestionarios $77.2 \%$, y en los crucigramas participaron $77.2 \%$ estudiantes. En la última semana de actividades en plataforma se realizó el postest Atención del niño y del adolescente enfermo.

El pretest del grupo experimental, lo respondieron 50 estudiantes, tuvieron un promedio de calificación en la escala de 1 al 10 de 6.04; en el grupo control respondieron 43 y obtuvieron un promedio de 6.83 , hay una diferencia de 0.79 puntos por encima del grupo experimental. (Ver tabla 1) 
Tabla 1. Resultados Pretest: "Atención del niño y del adolescente enfermo".

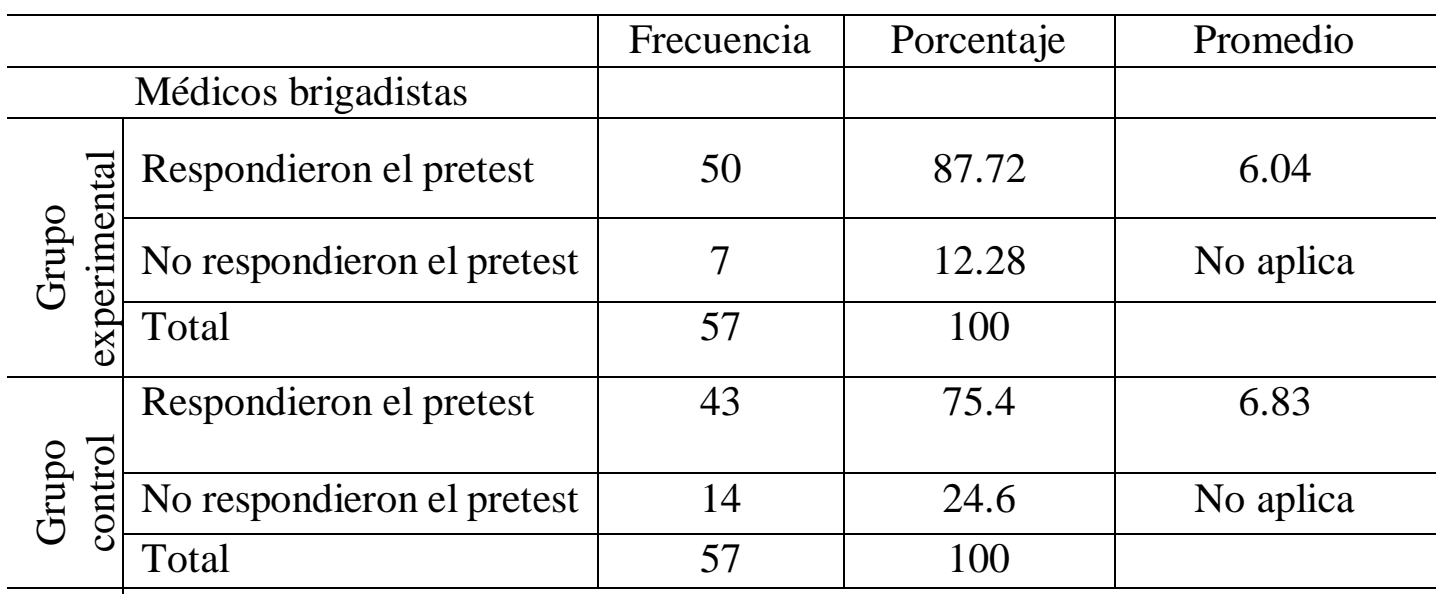

Elaboración propia (2019)

En el grupo experimental, 37 estudiantes realizaron el postest, tuvieron un promedio de 8.94; en el grupo control respondieron 30 y obtuvieron un promedio de 6.92 (Ver tabla 2). Entre el pretest y postest del grupo experimental hubo un aumento en el promedio de 2.9 puntos; en el grupo de control de 0.09 puntos.

Tabla 2. Resultados Postest: Atención del niño y del adolescente enfermo.

\begin{tabular}{|c|c|c|c|c|}
\hline \multirow{2}{*}{\multicolumn{2}{|c|}{ Médicos brigadistas }} & Frecuencia & Porcentaje & Promedio \\
\hline & & & & \\
\hline \multirow{3}{*}{ 율 } & $\begin{array}{l}\text { Respondieron el } \\
\text { postest }\end{array}$ & 37 & 87.72 & 8.94 \\
\hline & $\begin{array}{c}\text { No respondieron el } \\
\text { postest }\end{array}$ & 20 & 12.28 & No aplica \\
\hline & Total & 57 & 100 & \\
\hline \multirow{4}{*}{ 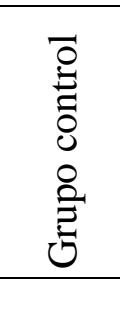 } & $\begin{array}{l}\text { Respondieron el } \\
\text { postest }\end{array}$ & 30 & 52.63 & 6.92 \\
\hline & $\begin{array}{l}\text { No respondieron el } \\
\text { postest }\end{array}$ & 27 & 47.37 & No aplica \\
\hline & Total & 57 & 100 & \\
\hline & \multicolumn{3}{|c|}{ Elaboración propia (2019) } & \\
\hline
\end{tabular}

Se puede apreciar en la tabla 1 y tabla 2 que la frecuencia de estudiantes del grupo experimental y grupo control que realizaron el pretest y postest no es el mismo. Entonces se eligieron sólo los que respondieron ambos y se realizó un comparativo. Del grupo 
experimental respondieron 35 estudiantes, en este grupo el promedio de calificación del pretest fue de 6 puntos, en el postest hubo un aumento de 3.97 puntos, obtuvieron un promedio de 8.97. El grupo de control respondieron 25, el promedio del pretest fue de 6.87 y el del postest de 6.96 (Ver tabla 3).

Tabla 3. Resultados de los médicos brigadistas que respondieron pretest y postest.

\begin{tabular}{|c|c|c|c|c|}
\hline & & Frecuencia & $\begin{array}{c}\text { Promedio } \\
\text { pretest }\end{array}$ & $\begin{array}{c}\text { Promedio } \\
\text { postest }\end{array}$ \\
\hline & Médicos brigadistas & & & \\
\hline ○ & $\begin{array}{l}\text { Respondieron pretest y } \\
\text { postest. }\end{array}$ & 35 & 6.00 & 8.97 \\
\hline$\underset{\overbrace{}}{\stackrel{0}{Z}}$ & $\begin{array}{l}\text { Respondieron pretest y } \\
\text { postest. }\end{array}$ & 25 & 6.87 & 6.96 \\
\hline
\end{tabular}

Para continuar el análisis de los datos, se realizó la prueba estadística $t$ de student para muestras relacionadas, debido a que es el mismo grupo evaluado (experimental) en 2 ocasiones (pretest y postest). En las muestras relacionadas se busca conocer si existe un incremento después de una intervención.

En el grupo experimental 35 estudiantes contestaron el instrumento (pretest y postest). La media del pretest fue de 6.00 y la del postest de 8.97. Al realizar el estadístico para pruebas relacionadas de $t$ de student con un nivel de significancia de 5\%, la estimación del p-valor fue de .000 , pertenece a la región de rechazo, por tanto, no hay evidencia suficiente en los datos muestrales para rechazar la hipótesis nula: Al implementar el programa académico de servicio social en modalidad blended-learning se incrementa el nivel de aprendizaje en los médicos brigadistas de servicio social por vinculación universitaria. (Ver tabla 4) 
Tabla 4. Prueba t de student para muestras relacionadas (pretest y postest del grupo experimental y grupo de control)

\begin{tabular}{l|l} 
& $\mathrm{p}$-valor \\
\hline $\begin{array}{l}\text { Médicos brigadistas que respondieron el } \\
\text { pretest y postest del grupo experimental }\end{array}$ & .000 \\
\hline $\begin{array}{l}\text { Médicos brigadistas que respondieron el } \\
\text { pretest y postest del grupo de control }\end{array}$ & .752 \\
\hline
\end{tabular}

Elaboración propia (2019)

Por otro lado, y para comparar el grupo experimental, se tuvo un grupo de control, la media del instrumento aplicado a este grupo en el pretest fue de $6.87 \mathrm{y}$ el postest de 6.96. Y el estadístico para pruebas relacionadas de t de student con un nivel de significancia de 5\%, la estimación del p-valor fue de .752 (pertenece a la región de aceptación), en donde se puede decir que el estudiante en la modalidad tradicional, sólo con su práctica de servicio social no mejoró el nivel de aprendizaje.

\section{Conclusiones}

Con lo anterior expuesto, se concluye que la implementación del programa académico de servicio social de medicina en la modalidad blended-learning (sesiones en presenciales con análisis de casos clínicos y sesiones en línea con recursos digitales como foros, tareas, crucigramas, cuestionarios, lecturas) mejoró el aprendizaje de los médicos brigadistas asignados a vinculación universitaria. El trabajo realizado corrobora el apoyo de integrar las TIC como estrategias y recursos didácticos al proceso educativo, ya que optimizó el aprendizaje.

El diseño del Aula Virtual crea un escenario de interacción y participación para los médicos brigadistas. Se sugiere establecer como obligatorio en las próximas generaciones de médicos brigadistas por vinculación universitaria. Además de contar con un mayor número de asesores de servicio social en la Facultad de Medicina, mediante la modalidad blendedlearning para garantizar la aplicación del programa académico de servicio social en su totalidad y para todos los brigadistas asignados a las unidades receptoras por vinculación universitaria. Así como tomar en cuenta esta investigación como referencia para su réplica en las diferentes facultades de medicina del país y de otras disciplinas del área de la salud. 


\section{Referencias}

Belloch, C. (2013). Diseño instruccional. Universidad de Valencia. Recuperado de: https://www.uv.es/bellochc/pedagogia/EVA4.pdf

Cáceres, M. (2015). Educación Online Superior y Abierta: ¿(R)evolución en la Formación Universitaria? Retos y Oportunidades de los MOOCs (Massive Open online Courses) para la Formación Superior Europea en la Sociedad de la Información. Tesis doctoral. Universidad Nacional de Educación a Distancia (UNED), Madrid, España.

Cobo, C. (2009). El concepto de tecnologías de la información. Benchmarking sobre las definiciones de las TIC en la sociedad del conocimiento. Zer: Revista de estudios de comunicación = Komunikazio ikasketen aldizkaria, ISSN 1137-1102, №. 27, 2009, pags. 295-318. 14.

Coll, C., \& Monereo, C. (2008). Educación y aprendizaje en el siglo XXI Nuevas herramientas, nuevos escenarios,nuevas finalidades. En Coll, C., Monereo, C. (eds.), Psicología de la educación virtual. Madrid: Morata.

Coll, C., Mauri, T \& Onrubia, J. (2008). La utilización de las tecnologías de la información y la comunicación en la educación: del diseño tecno-pedagógico a las prácticas de uso. En Coll, C., Monereo, C. (eds.), Psicología de la educación virtual. Madrid: Morata.

López de la Serna, A. (2016). Integración de los MOOC en la enseñanza universitaria. El caso de los SPOC. Tesis doctoral. Universidad del País Vasco / EuskalHerrikoUnibertsitatea, Leioa, España.

Martínez-Fernández, J. R., \& Rabanaque, S. (2008). Autorregulación y trabajo autónomo del estudiante en una actividad de aprendizaje basada en las TIC. Anuario de Psicología, 39(3),311-331. [fecha de Consulta 26 de febrero de 2020]. ISSN: 0066-5126. Disponible en : https://www.redalyc.org/articulo.oa?id=970/97021031002 
Mauri, T \& Onrubia, J. (2008). El profesor en entornos virtuales: Condiciones, perfil y competencias. En Coll, C., Monereo, C. (eds.) Psicología de la educación virtual. Madrid: Morata.

Mirete, R., García, S., \& Hernández, P. (2015). Cuestionario para el estudio de la actitud, el conocimiento y el uso de TIC (ACUTIC) en Educación Superior. Estudio de fiabilidad y validez. Revista interuniversitaria de formación del profesorado., (83), 75-89. Recuperado de: https://www.redalyc.org/pdf/274/27443659006.pdf

Pérez-Tejeda, H. E. (2008). Estadística para las ciencias sociales, del comportamiento y de la salud. Cenjage Learning: México.

Preza, M., \& Hernández, C. (2015). Instrumento para Medir la Satisfacción de un Curso de Formación Directiva en Modalidad B-Learning. EDUCATECONCIENCIA, 7(8). Recuperado de: http://tecnocientifica.com.mx/educateconciencia/index.php/revistaeducate/article/vi ewFile/34/29

Soto, M. G. (2014). Aprendizaje Significativo y Blended-learning: Construcción de un paradigma de integración didáctico-tecnológico en educación superior. (Tesis de Doctorado). Universidad Autónoma de Sinaloa, Culiacán Sinaloa, México. 